\title{
DIRECT COMPUTATION OF THE SPECTRAL FUNCTION
}

\author{
AMIN BOUMENIR
}

(Communicated by Palle E. T. Jorgensen)

\begin{abstract}
We would like to find an explicit formula for the spectral function of the following Sturm-Liouville problem:

$$
\left\{\begin{array}{l}
L f \equiv-\frac{d^{2}}{d x^{2}} f(x)+q(x) f(x), \quad x \geq 0, \\
f^{\prime}(0)-m f(0)=0 .
\end{array}\right.
$$

A simple operational calculus argument will help us obtain an explicit formula for the transmutation kernel. The expression of the spectral function is then obtained through the nonlinear integral equation found in the Gelfand-Levitan theory.
\end{abstract}

\section{INTRODUCTION}

Some forty years ago, Gelfand and Levitan, in a celebrated article [3] obtained an integral equation that related the spectral function $\Gamma(\lambda)$ of the selfadjoint operator

$$
\left\{\begin{array}{l}
L f \equiv-\frac{d^{2}}{d x^{2}} f(x)+q(x) f(x), \quad x \geq 0, \\
f^{\prime}(0)-m f(0)=0
\end{array}\right.
$$

to the real function $q(x)$ and $m$. The purpose of this note is not to expose the work in [3] but to work out a method that gives the spectral function $\Gamma(\lambda)$ explicitly in terms of the potential $q(x)$, i.e. solve the direct spectral problem. The importance of the spectral function lies in the fact that it contains all information about the spectrum of the operator. Indeed, the decomposition of the spectrum will correspond to the decomposition of the measure.

Recall that with each selfadjoint operator is associated a unitary transform by which the operator is transformed into a simple multiplication by the independent variable. In our case this transform is represented by

$$
\begin{aligned}
L_{d x}^{2}(0, \infty) & \rightarrow L_{d \Gamma(\lambda)}^{2}(0, \infty) \\
f & \rightarrow \hat{f}(\lambda) \equiv \int_{0}^{\infty} f(x) \overline{y(x, \lambda)} d x .
\end{aligned}
$$

Received by the editors May 2, 1994.

1991 Mathematics Subject Classification. Primary 47A11.

(C)1995 American Mathematical Society 
The inverse transform is simply defined by $f(x) \equiv \int \hat{f}(\lambda) y(x, \lambda) d \Gamma(\lambda)$ where $\Gamma(\lambda)$ is the spectral function and $y(x, \lambda)$ are solutions of

$$
\left\{\begin{array}{l}
-y^{\prime \prime}+q y=\lambda y \\
y(0, \lambda)=1 \text { and } y^{\prime}(0, \lambda)=m
\end{array}\right.
$$

i.e., eigenfunctionals of $L$. The theory of the inverse spectral problem is based on the existence of two functions $K(x, t), H(x, t)$ such that

$$
\left\{\begin{array}{l}
y(x, \lambda)=\cos (x \sqrt{\lambda})-\int_{0}^{x} K(x, t) \cos (t \sqrt{\lambda}) d t \quad \forall \lambda \in R, \\
\cos (x \sqrt{\lambda})=y(x, \lambda)-\int_{0}^{x} H(x, t) y(t, \lambda) d t .
\end{array}\right.
$$

By a process of elimination one obtains the well-known linear integral equation in $K(x, t)$ and $H(x, t)$ derived by Gelfand and Levitan. With the help of the function $H(x, t)$ one can express the spectral function (see [3]), through what is called the nonlinear equation,

(3) $\int \cos (x \sqrt{\lambda}) \cos (t \sqrt{\lambda}) d \sigma(\lambda)=-H(x, t)-H(t, x)+\int_{0}^{x} H(x, s) H(s, t) d s$

where

$$
\sigma(\lambda) \equiv \begin{cases}\Gamma(\lambda)-\frac{2}{\pi} \sqrt{\lambda} & \text { for } \lambda \geq 0 \\ \Gamma(\lambda) & \text { for } \lambda<0\end{cases}
$$

In fact we can obtain a simpler and remarkable expression, if we assume that $\Gamma(\lambda)$ is absolutely continuous with respect to $d \sqrt{\lambda}$ (see [2])

$$
\frac{\pi}{2} \frac{d \Gamma(\lambda)}{d \sqrt{\lambda}}=1-\int_{0}^{\infty} H(t, 0) \cos (t \sqrt{\lambda}) d t, \quad \lambda>0 .
$$

Our first objective is to obtain an explicit formula for the function $H(x, t)$ which can be used in the above expression to obtain $\Gamma(\lambda)$. Recall that the functions $K(x, t)$ and $H(x, t)$ are defined in fact through partial differential equations of hyperbolic type, and this alone makes it impossible to obtain $H(x, t)$ explicitly. In this note we shall derive the function $H(x, t)$ in a direct and natural way. It remains to use the inverse cosine transform in (3) to recover the expression of the spectral function $\Gamma(\lambda)$.

\section{EXPLicit COMPUTATION OF $H(x, t)$}

In this section we shall try to compute explicitly the kernel $H(x, t)$ appearing in equation (2). For the sake of simplicity we first deal with the case $m=0$. Equation (1) can be rewritten as an integral equation

$$
y(x, \lambda)=\cos (x \sqrt{\lambda})+\int_{0}^{x} \frac{\sin ((x-t) \sqrt{\lambda})}{\sqrt{x}} \cdot q(t) y(t, \lambda) d t .
$$

From this and equation (2) one obtains

$$
\int_{0}^{x} H(x, t) y(t, \lambda) d t=\int_{0}^{x} \frac{\sin ((x-t) \sqrt{\lambda})}{\sqrt{\lambda}} \cdot q(t) y(t, \lambda) d t
$$

It is readily seen that in order to recover the function $H(x, t)$ we need to write the right-hand side of (5) as a $y$-transform. Hence our first objective is to get 
rid of the $\lambda$ appearing in $\frac{\sin ((x-t) \sqrt{\lambda})}{\sqrt{\lambda}}$ by converting it to an operator. Observe that since the kernel is an entire function,

$$
\frac{\sin ((x-t) \sqrt{\lambda})}{\sqrt{\lambda}} \equiv \sum_{n \geq 0}(-1)^{n} \frac{(x-t)^{2 n+1}}{(2 n+1) !} \lambda^{n},
$$

the uniform convergence in $[0, x]$ implies

$$
\int_{0}^{x} H(x, t) y(t, \lambda) d t=\sum_{n \geq 0} \int_{0}^{x} \frac{(x-t)^{2 n+1}}{(2 n+1) !}(-1)^{n} q(t) \lambda^{n} y(t, \lambda) d t .
$$

For simplicitly we shall agree to write

$$
\mathscr{L} \equiv-\frac{d^{2}}{d t^{2}}+q(t) \text { and } a_{n}(x-t) \equiv \frac{(x-t)^{2 n+1}}{(2 n+1) !}(-1)^{n} .
$$

Recall in this case $\mathscr{L}^{n} y(x, \lambda)=\lambda^{n} y(x, \lambda)$ holds in the classical sense and therefore (6) yields

$$
\int_{0}^{x} H(x, t) y(t, \lambda) d t=\sum_{n \geq 0} \int_{0}^{x} a_{n}(x-t) q(t) \mathscr{L}^{n} y(t, \lambda) d t .
$$

To simplify the integration by parts, let us assume that

$$
q(t) \in \mathscr{C}^{\infty}[0, \infty) \quad \text { and } \quad q^{(k)}(0)=0 \quad \forall k \geq 0 .
$$

Clearly for $n \geq m$

$$
\begin{aligned}
\mathscr{L}^{m} a_{n}(x-t) q(t) & =\left(q(t)-\frac{d^{2}}{d t^{2}}\right)^{m}\left[\frac{(x-t)^{2 n+1}}{(2 n+1) !(-1)^{n}} q(t)\right] \\
& =\sum c_{\beta, r, s} q(t)^{\beta} q(t)^{(r)}(x-t)^{s}
\end{aligned}
$$

where $2 n \geq r, \beta \geq 1$, and $s \geq 1$. Thus the contribution of the boundary condition at $t=0$ and $t=x$ is zero since $q^{(k)}(0)=0, k \geq 0$, and $s \geq 1$. For each fixed $x$, obviously $\frac{d^{2 n}}{d t^{2 n}}(x-t)^{(2 n+1)}$ is continuous at $t=x$. Consequently $\left[q(t)-\frac{d^{2}}{d t^{2}}\right]^{n}(x-t)^{(2 n+1)} q(t)$ is also continuous in $[0, x]$.

From (7) we deduce that

$$
\int_{0}^{x} H(x, t) y(t, \lambda) d t=\sum_{n \geq 0} \int_{0}^{x} \mathscr{L}^{n}\left[a_{n}(x-t) q(t)\right] y(t, \lambda) d t .
$$

Remark. From the Gelfand-Levitan theory, $H(x, \cdot) \in \mathscr{C}^{\infty}[0, x]$ and so the left-hand side is $L_{d \Gamma(\lambda)}^{2}$. This means that, for each fixed $x$, the series on the right converges in $L_{d \Gamma(\lambda)}^{2}$ and obviously the partial sums also belong to $L_{d \Gamma(\lambda)}^{2}$, in other words,

$$
\sum_{k=0}^{n}(-1)^{k} \lambda^{k} \int_{0}^{x} \frac{(x-t)^{2 k+1}}{(2 k+1) !} q(t) y(t, \lambda) d t \in L_{d \Gamma(\lambda)}^{2} .
$$

The next Lemma will help us interchange the series with the integral in (8). 
Lemma. $\sum_{k=0}^{k=n} \mathscr{L}^{k}\left[a_{k}(x-t) q(t)\right]=\mathscr{L}^{n+1} a_{n}(x-t)$.

Proof. It is readily seen that

$\mathscr{L}^{k+1} a_{k}(x-t)=\mathscr{L}^{k}\left[\left(q(t)-D^{2}\right) a_{k}(x-t)\right]=\mathscr{L}^{k}\left[\left(q(t) a_{k}(x-t)\right]+\mathscr{L}^{k}\left[a_{k-1}(x-t)\right]\right.$.

Hence

$$
\begin{aligned}
\sum_{k=0}^{k=n} \mathscr{L}^{k}\left[a_{k}(x-t) q(t)\right] & =a_{0}(x-t) q(t)+\sum_{k=1}^{k=n} \mathscr{L}^{k+1} a_{k}(x-t)-\mathscr{L}^{k}\left[a_{k-1}(x-t)\right] \\
& =a_{0}(x-t) q(t)+\mathscr{L}^{n+1} a_{n}(x-t)-\mathscr{L}\left[a_{0}(x-t)\right] \\
& =\mathscr{L}^{n+1} a_{n}(x-t) .
\end{aligned}
$$

Hence the Lemma is proved and as a consequence

$$
\sum_{k=0}^{k=n} \int_{0}^{x} \mathscr{L}^{k}\left[a_{k}(x-t) q(t)\right] y(t, \lambda) d t=\int_{0}^{x} \mathscr{L}^{n+1}\left[a_{n}(x-t)\right] y(t, \lambda) d t .
$$

Since the partial sum converges in $L_{d \Gamma(\lambda)}^{2}$, we have

$$
\int_{0}^{x} \mathscr{L}^{n+1}\left[a_{n}(x-t)\right] y(t, \lambda) d t \stackrel{L_{d \Gamma(\lambda)}^{2}}{\longrightarrow} \int_{0}^{x} H(x, t) y(t, \lambda) d t \quad \text { as } n \rightarrow \infty .
$$

Using the inverse $y$-transform, which is a unitary transform, we deduce

$$
\forall x, \quad \mathscr{L}^{n+1}\left[a_{n}(x-t)\right] \stackrel{L_{d t}^{2}(0, x)}{\longrightarrow} H(x, t) \text { as } n \rightarrow \infty .
$$

Thus we have proved

Proposition 1. Let $q(t) \in \mathscr{C}^{\infty}, q^{(n)}(0)=0 \forall n \geq 0, \forall x>0$ and $0 \leq t \leq x$; then

$$
H(x, t) \equiv \lim _{n \rightarrow \infty}\left[-\frac{d^{2}}{d t^{2}+q(t)}\right]^{n+1}\left\{\frac{(-1)^{n}(x-t)^{2 n+1}}{(2 n+1) !}\right\} \quad \text { in } L_{d t}^{2}(0, x) .
$$

Remark. In other words, since $H(x, t)$ is the kernel of a transmutation, we have

$$
\cos (x \sqrt{\lambda})=y(x, \lambda)-\lim _{n \rightarrow \infty} \int_{0}^{x}\left[-\frac{d^{2}}{d t^{2}}+q(t)\right]^{n+1}\left\{\frac{(-1)^{n}(x-t)^{2 n+1}}{(2 n+1) !}\right\} y(t, \lambda) d t .
$$

The above expression is the key to our problem. Indeed as mentioned before we do not need to solve a partial differential equation in order to find $H(x, t)$. We can also repeat a similar argument to obtain the function $K(x, t)$.

\section{THE SPECTRAL FUNCTION}

Now we are in a position to find the spectral function just by using the nonlinear equation (3) which can also be obtained as a particular case from [1].

Proposition 2. Let $q(x) \in \mathscr{C}^{\infty}$ and $q^{(n)}(0)=0$, and $\Gamma\left(\lambda^{2}\right)$ be differentiable and $\operatorname{supp} d \Gamma(\lambda) \subset[0, \infty)$. Then

$$
\frac{\pi}{2} \frac{d \Gamma(\lambda)}{d \sqrt{\lambda}} \equiv 1-\int_{0}^{\infty} H(t, 0) \cos (t \sqrt{\lambda}) d t
$$


where

$$
H(x, t) \equiv \lim _{n \rightarrow \infty}\left[-\frac{d^{2}}{d t^{2}}+q(t)\right]^{n+1}\left\{\frac{(-1)^{n}(x-t)^{2 n+1}}{(2 n+1) !}\right\} .
$$

Proof. From the assumptions that $\Gamma\left(\lambda^{2}\right)$ is differentiable and supp $d \Gamma(\lambda) \subset$ $[0, \infty),(3)$ can be written as

$$
\begin{aligned}
& \int \cos (x \sqrt{\lambda}) \cos (t \sqrt{\lambda})\left[\frac{\pi}{2} \frac{d \Gamma(\lambda)}{d \sqrt{\lambda}}-1\right] d \frac{2}{\pi} \sqrt{\lambda} \\
& =-H(x, t)-H(t, x)+\int_{0}^{x} H(x, s) H(s, t) d s
\end{aligned}
$$

By taking the inverse cosine transform, it follows that for each $x$, in the space $L_{d \sqrt{\lambda}}^{2}$

$$
\begin{aligned}
\left(\frac{\pi}{2} \frac{d \Gamma}{d \sqrt{\lambda}}(\lambda)-1\right) \cos (x \sqrt{\lambda}) \\
\equiv-\int_{0}^{x} H(x, t) \cos (t \sqrt{\lambda}) d t-\int_{x}^{\infty} H(t, x) \cos (t \sqrt{\lambda}) d t \\
\quad+\int_{0}^{x} H(x, t) \int_{t}^{\infty} H(\eta, t) \cos (\eta \sqrt{\lambda}) d \eta d t
\end{aligned}
$$

By letting $x \rightarrow 0$ we obtain the proposition.

Remark. The connection between the spectral function and the kernel $H(x, t)$ is a standard result (see [2]). However the assumption on the spectral function simplifies the proof.

\section{GeNERAL BOUNDARY CONDITION}

We now consider the general boundary condition, i.e. $L$ is a selfadjoint extension defined by

$$
\left\{\begin{array}{l}
L f=-\frac{d^{2} f}{d x^{2}}+q(x) f, \\
f^{\prime}(0)-m f(0)=0, \quad m \in R
\end{array}\right.
$$

where $m \neq 0$. The eigenfunctionals in this case are defined by

$$
\left\{\begin{array}{l}
-y^{\prime \prime}(x, \lambda)+q(x) y(x, \lambda)=\lambda y(x, \lambda) \\
y(0, \lambda)=1 \text { and } y^{\prime}(0, \lambda)=m
\end{array}\right.
$$

and the solution of the above equation can be written as

$$
y(x, \lambda)=\cos (x \sqrt{\lambda})+m \frac{\sin (x \sqrt{\lambda})}{\sqrt{\lambda}}+\int_{0}^{x} \frac{\sin ((x-t) \sqrt{\lambda})}{\sqrt{\lambda}} \cdot q(t) y(t, \lambda) d t
$$

Under conditions of Proposition 1, we can also integrate by parts to obtain $y(t, \lambda)=\cos (x \sqrt{\lambda})+m \frac{\sin (x \sqrt{\lambda})}{\sqrt{\lambda}}+\int_{0}^{x} \lim _{n \rightarrow \infty} \mathscr{L}^{n+1}\left\{\frac{(x-t)^{2 n+1}(-1)^{n}}{(2 n+1) !}\right\} y(t, \lambda) d t$.

If we denote by $L_{2, m}$ the selfadjoint extension defined by

$$
\left\{\begin{array}{l}
L_{2, m} f \equiv-f^{\prime \prime}(x), \quad x>0, \\
f^{\prime}(0)-m f(0)=0,
\end{array}\right.
$$


then its spectral function

$$
\text { if } m \geq 0 \quad \frac{d \Gamma_{2, m}}{d \lambda} \equiv \begin{cases}\frac{\sqrt{\lambda}}{\pi\left(\lambda+m^{2}\right)}, & \lambda \geq 0 \\ 0, & \lambda<0\end{cases}
$$

and

$$
\text { if } m<0 \quad \frac{d \Gamma_{2, m}}{d \lambda} \equiv \begin{cases}\frac{\sqrt{\lambda}}{\pi\left(\lambda+m^{2}\right)}, & \lambda \geq 0, \\ -2 m \delta\left(\lambda+m^{2}\right), & \lambda<0,\end{cases}
$$

By using the factorization theorem (see [1]), and repeating the proof of Proposition 2, we end up with

$$
\frac{d \Gamma}{d \Gamma_{2, m}}(\lambda)=1-\int_{0}^{\infty} H(t, 0)\left[\cos (t \sqrt{\lambda})+m \frac{\sin (t \sqrt{\lambda})}{\sqrt{\lambda}}\right] d t \quad \text { for } \lambda>0
$$

Proposition 3. Let $q(t) \in \mathscr{C}^{\infty}$ and $q^{(n)}(0)=0 \forall n \geq 0$, and let $\Gamma(\lambda)$ be absolutely continuous with respect to $d \Gamma_{2, m}(\lambda)$. Then

$$
\frac{d \Gamma}{d \Gamma_{2, m}}(\lambda)=1-\int_{0}^{\infty}\left(\cos (t \sqrt{\lambda})+m \frac{\sin (t \sqrt{\lambda})}{\sqrt{\lambda}}\right) H(t, 0) d t
$$

where

$$
H(x, t) \equiv \lim _{n \rightarrow \infty}\left[-\frac{d^{2}}{d t^{2}}+q(t)\right]^{n+1}\left\{\frac{(-1)^{n}(t-x)^{2 n+1}}{(2 n+1) !}\right\}
$$

\section{ACKNOWLEDGMENT}

The author is indebted to K.F.U.P.M. for its support.

\section{REFERENCES}

1. A. Boumenir, Comparison theorem for self-adjoint operators, Proc. Amer. Math. Soc. 111 (1991), 161-175.

2. R. W. Carroll, Transmutations, scattering theory and special functions, North-Holland, Math. Stud., vol. 69, North-Holland, Amsterdam and New York, 1982.

3. I. M. Gelfand and B. M. Levitan, On the determination of a differential equation from its spectral function, Amer. Math. Soc. Transl. Ser. 21 (1951), 239-253.

Department of Mathematics, King Fahad University of Petroleum and Minerals, Dhahran, Saudi ARABia

Current address: Department of Mathematics, University of Delaware, Newark, Delaware 19716-2553

E-mail address: fac10460saupm00.bitnet 